UDC: $517.55: 514.181 .4$

45 (LXXI) No.1 (2021), 17-21

DOI: $10.37560 /$ matbil21451017h

\title{
ON THE BOREL THEOREM
}

\author{
FATHI HAGGUI ${ }^{1}$ AND ABDESSAMI JALLED ${ }^{2}$
}

\begin{abstract}
In this note we give a new characterization of holomorphic curves which lies in the complement of $2 n$ hyperplanes in general position. This is a generalisation of The Borel Theorem. By M.Green, any holomorphic curves which lies in the complement of $2 n+1$ hyperplanes in general position is constant. Using our generalisation of The Borel Theorem, we can easily show Green's Theorem.
\end{abstract}

\section{INTRODUCTION}

The classical Picard Theorem [8] (see also [6] and [11]) states that every holomorphic map from the complex Euclidean space $\mathbb{C}$ to $\mathbb{C} P^{1}$ that omits three points, is constant. This Theorem has been extended to higher dimension by M.Green [9] (see also [2] and [7]), who provided examples of complex Kobayashi hyperbolic manifolds. We recall that given a complex manifold $X$, then $X$ is said to be Kobayashi hyperbolic if the following kobayashi-Royden pseudo-metric is non degenerate:

$$
K_{X}(p, v):=\inf \left\{\alpha>0 ; \exists f: \mathbb{D} \rightarrow X \text { holomorphic, } f(0)=p, f^{\prime}(0)=\frac{v}{\alpha}\right\} .
$$

With $p \in X$ and $v \in T_{p}(X)$.

We call a complex manifold $X$ Brody hyperbolic (B-hyperbolic) if any holomorphic curve $f: \mathbb{C} \rightarrow X$ is constant. Obviously, Kobayashi hyperbolicity implies B-hyperbolicity. The reverse is true for compact complex manifolds [3]. There are many examples of compact complex manifolds that are hyperbolic according to Kobayashi, and so by Brody Theorem (see [3]), have the prorerty that each holomorphic curve $f: \mathbb{C} \rightarrow X$ is constant. We recall that if $H_{1}, \ldots, H_{m}$ are complex hyperplanes in $\mathbb{C} P^{n}$, then they are said to be in general position if $m \geqslant n+1$ and any $(n+1)$ of these hyperplanes are linearly independent. Since Bloch and Cartan, the hyperbolicity of the complement of arrangements of projective lines in general position in the complex projective plane has been the subject of numerous

2010 Mathematics Subject Classification. Primary: 32Q45, 52C35, 32H02, 32S22.

Key words and phrases. Complex manifold, Projective space, Holomorphic curves, Holomorphic curves omitting hyperplane, Kobayashi hyperbolicity. 
studies for many years and by several researchers (as Mikhail Zaidenberg, Adel Khalfallah and Hervé Gaussier). Different results were obtained for some special cases, especially the next Theorem due to Borel, stated by Cartan in the following form (see [4], [2] and also [1]):

Theorem 1 (Borel Theorem). Let $L_{1}, L_{2}, L_{3}$ and $L_{4}$ be four projective lines of $\mathbb{C} P^{2}$ in the general position, that is, such that the configuration $C=L_{1} \cup L_{2} \cup L_{3} \cup L_{4}$ does not have a triple point. Let us note $\Delta$ be the union of the three diagonals $\Delta_{1}, \Delta_{2}$ and $\Delta_{3}$ of the configuration $C$, that is the projective lines passing through double points of $C$. Then, any non-constant entire curve with values in $\mathbb{C} P^{2} \backslash C$ degenerates in $\Delta$. (i.e there exists $i \in\{1,2,3\}$ such that $\left.f(\mathbb{C}) \subset \Delta_{i}\right)$.

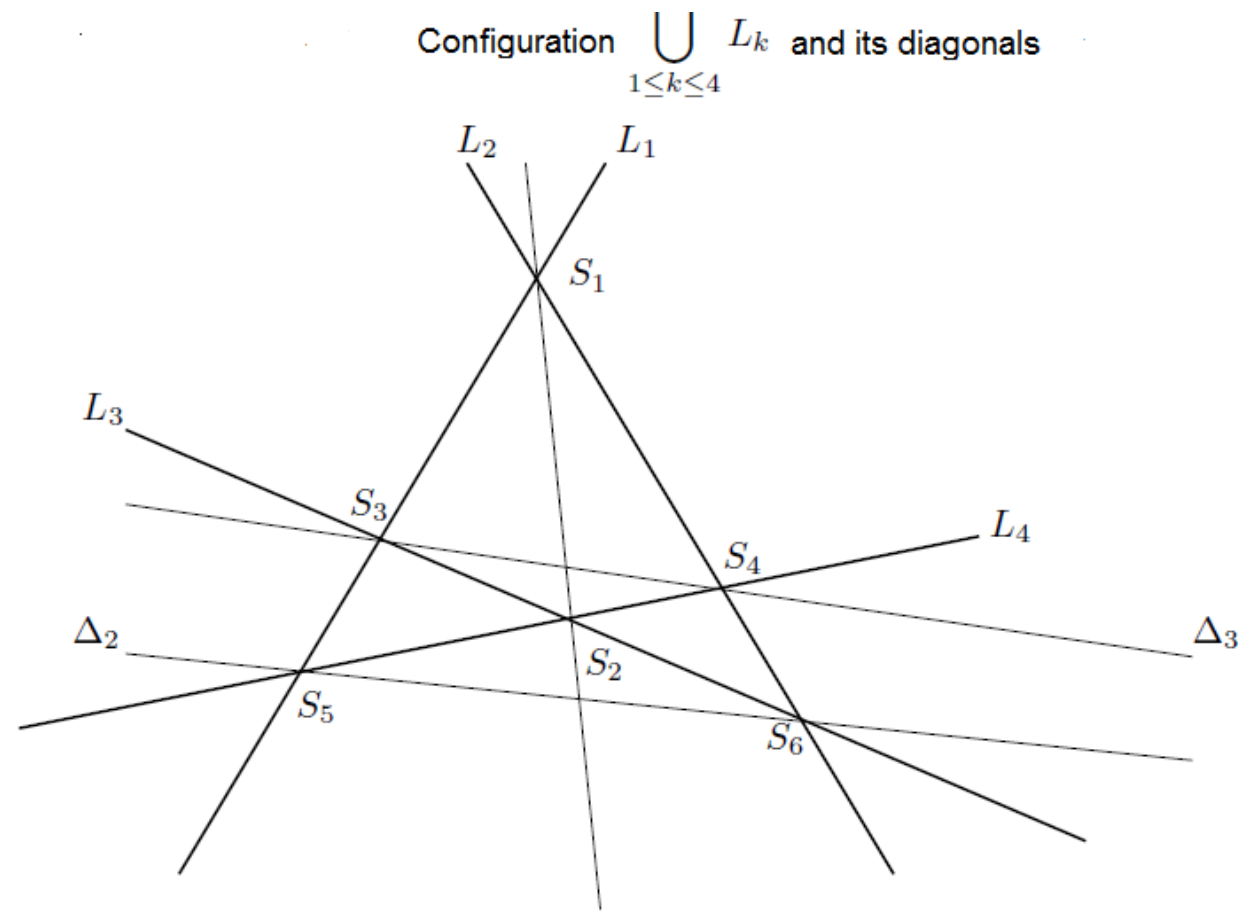

$\Delta_{1}$

Thus, via R. Brody's reparameterization Theorem (see [3], [5] and also [1]), M. Green was able to deduce from Borel's theorem the hyperbolically embedded character of the complement of five lines in the projective plane. He showed the following:

Theorem 2. [9], [11]

Let $H_{1}, H_{2}, \ldots, H_{2 n+1}$ be $2 n+1$ hyperplanes in general position in $\mathbb{C} P^{n}$. Then any holomorphic curves $f: \mathbb{C} \rightarrow \mathbb{C} P^{n} \backslash \cup_{i=1}^{2 n+1} H_{i}$ is constant.

The main goal in this work is to generalize the Borel Theorem. We show the following: 
Theorem 3. Let $H_{1}, \ldots, H_{2 n}$ be $(2 n)$ projective hyperplanes in general position in $\mathbb{C} P^{n}$. Then there are $\frac{1}{2} C_{2 n}^{n}$ diagonals $\Delta_{1}, \ldots ., \Delta_{\frac{1}{2} C_{2 n}^{n}}$ such that for any non constant holomorphic curve $f: \mathbb{C} \longrightarrow \mathbb{C} P^{n} \backslash \cup_{i=1}^{2 n} H_{i}$, there exists $k_{f} \in\left\{1, \ldots, \frac{1}{2} C_{2 n}^{n}\right\}$ such that $f(\mathbb{C}) \subset \Delta_{k_{f}}$.

1.1. The Fujimoto Theorem. In 1972, Fujimoto [10] (see also M.Green[9]) showed a statement that characterizes the image of a holomorphic map $f: \mathbb{C} \rightarrow \mathbb{C} P^{n}$ omitting $(n+p)$ hyperplanes in general position. He proved the following:

Theorem (Serge Lang [10] p.196). Let $f: \mathbb{C} \rightarrow \mathbb{C} P^{n}$ be holomorphic. Assume that the image of $f$ lies in the complement of $n+p$ hyperplanes in general position, then this image is contained in a complex projective subspace of complex dimension $\leqslant[n / p]$, where $[$.$] denotes the greatest integer.$

The version of the Green Theorem stated in the introduction is a particular case of the previous Theorem, with $p=n+1$.

\section{Proof of Theorems 3}

Definition 2.1. Let $H_{1}, \ldots, H_{m}, m \geqslant 2 n$, be hyperplanes in general position of $\mathbb{C} P^{n}$. We call diagonal, the line passing through the two points $\cap_{i \in I} H_{i}$ and $\cap_{j \in J} H_{j}$, where $|I|=|J|=n$ and $I \cap J=\emptyset$. Here $|I|$ denotes the cardinal of $I$.

Proof. (of Theorem 3). The proof is inspired by the Fujimoto Theorem, (see [10]).

Let $f: \mathbb{C} \longrightarrow \mathbb{C} P^{n}$ be holomorphic, such that $f(\mathbb{C}) \cap\left(\cup_{i=1}^{2 n} H_{i}\right)=\emptyset$.

Let $L_{1}, \ldots, L_{2 n}$ be linear forms defining the hyperplanes $H_{1}, \ldots, H_{2 n}$, namely $H_{k}=$ $L_{k}^{-1}(\{0\})$ for $k=1, \ldots, 2 n$. If $f=\left[f_{1}: \ldots: f_{n+1}\right]$, we denote

$$
h_{k}:=L_{k}(f), k=1, \ldots, 2 n .
$$

Let $I=\{1, \cdots, 2 n\}$ be the set of indices and $\sim$ be the equivalence relation defined by $i \sim j$ if $h_{i} / h_{j}$ is constant. We take a partition of the set of indices according to $\sim$. First, we know that the complement of a given class $S$ has at most $n$ elements. Hence $S$ has at least $n$ elements and there are at most two classes.

The case of one class is not possible. Indeed, suppose that there exists $\alpha_{2}, \ldots, \alpha_{2 n} \in \mathbb{C}$ such that

$$
\text { (S) }\left\{\begin{aligned}
h_{2} & =\alpha_{2} h_{1} \\
h_{3} & =\alpha_{3} h_{1} \\
& \vdots \\
h_{2 n} & =\alpha_{2 n} h_{1}
\end{aligned}\right.
$$

The first equation $h_{2}=\alpha_{2} h_{1}$ implies that $h_{2}-\alpha_{2} h_{1}=0$. Since, by construction, $h_{k}:=L_{k}(f)$ then we obtain:

$$
\begin{aligned}
& L_{2}(f)-\alpha_{2} L_{1}(f)=0 \\
\Rightarrow \quad & \left(L_{2}-\alpha_{2} L_{1}\right)(f)=0 \\
\Rightarrow \quad & f(\mathbb{C}) \subset \operatorname{ker}\left(L_{2}-\alpha_{2} L_{1}\right)
\end{aligned}
$$


Now, since $L_{2}-\alpha_{2} L_{1}$ is a combination of two linear forms defining the hyperplanes $H_{2}$ and $H_{1}$, then

$$
f(\mathbb{C}) \subset \operatorname{ker}\left(L_{2}-\alpha_{2} L_{1}\right) \Longrightarrow f(\mathbb{C}) \subset H_{2} \cap H_{1}
$$

This is for the first equation. By doing the same for the other equations, we obtain

$$
f(\mathbb{C}) \subset \cap_{k=2}^{n+1}\left(H_{k} \cap H_{1}\right)=\cap_{k=1}^{n+1} H_{k}=\emptyset,
$$

which is impossible. Hence there are exactly two classes $S_{1}$ and $S_{2}$.

We know that each of the two classes $S_{1}$ and $S_{2}$ contains $n$ elements. Then there exists a permutation $\sigma:\{1, \ldots, 2 n\} \rightarrow\{1, \ldots, 2 n\}$ such that

$$
S_{1}=\{\sigma(1), \ldots, \sigma(n)\}, S_{2}=\{\sigma(n+1), \ldots, \sigma(2 n)\}
$$

Hence, there exist $\alpha_{2}, \ldots, \alpha_{n}, \beta_{n+1}, \ldots, \beta_{2 n-1} \in \mathbb{C}$ such that $h_{1}, \ldots, h_{2 n}$ satisfy the systems:

$$
\left(S_{1}\right)\left\{\begin{array} { r l } 
{ h _ { \sigma ( 2 ) } } & { = \alpha _ { 2 } h _ { \sigma ( 1 ) } } \\
{ h _ { \sigma ( 3 ) } } & { = \alpha _ { 3 } h _ { \sigma ( 1 ) } } \\
{ } & { \vdots }
\end{array} \quad ( S _ { 2 } ) \left\{\begin{array}{rl}
h_{\sigma(n+1)} & =\beta_{n+1} h_{\sigma(2 n)} \\
h_{\sigma(n+2)} & =\beta_{n+2} h_{\sigma(2 n)} \\
h_{\sigma(n)} & =\alpha_{n} h_{\sigma(1)}
\end{array}\right.\right.
$$

Hence, by following the same procedure done in the previous system, we get

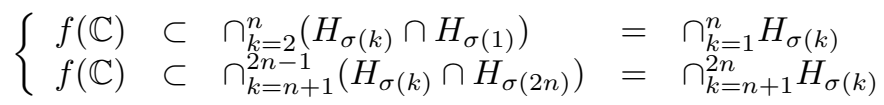

Then $f(\mathbb{C}) \subset \Delta_{\sigma}$, where $\Delta_{\sigma}$ is the unique diagonal (line) passing through the two points $\cap_{k=1}^{n} H_{\sigma(k)}$ and $\cap_{k=n+1}^{2 n} H_{\sigma(k)}$.

Now the two points, and consequently $\Delta_{\sigma}$, are completely determined by $S_{1}=\{\sigma(1), \ldots, \sigma(n)\}$ since $S_{2}$ is automatically fixed once $S_{1}$ is chosen. Hence $\Delta_{\sigma}$ is completely determined by a choice of a partition of $\{1, \ldots, 2 n\}$ into two subsets, each of them containing $n$ elements. There are exactly $\frac{1}{2} C_{2 n}^{n}$ such partitions. This proves the Theorem.

Corollary 3.1. Any holomorphic curve that lies in the complement of $2 n+1$ hyperplanes in general position in $\mathbb{C} P^{n}$, is constant.

Proof. Since $f(\mathbb{C})$ omits $\cup_{i=1}^{2 n+1} H_{i}$, then in particular $f(\mathbb{C})$ omits $\cup_{i=1}^{2 n} H_{i}$. Hence by Theorem 3 there exists a diagonal $\Delta_{1}$ such that $f(\mathbb{C}) \subset \Delta_{1}$. On the other hand, since $f(\mathbb{C})$ omits $\cup_{i=2}^{2 n+1} H_{i}$, then there exists $\Delta_{2}$ such that $f(\mathbb{C}) \subset \Delta_{2}$. Hence

$$
f(\mathbb{C}) \subset \Delta \cap \Delta^{\prime}=\{p t\}
$$

Thus $f$ is constant. 
Acknowledgement: The authors would like to express their sincere appreciation for the valuable suggestions and informative comments provided by the reviewers of the manuscript.

\section{REFERENCES}

[1] S. Benoit, Trois problèmes géométriques d'hyperbolicité complexe et presque complexe, Diss. Université Paris Sud-Paris XI, 2011

[2] S. Benoit, Un théorème de Bloch presque complexe, Annales de l'Institut Fourier, 2014, p. 401-428.

[3] R. Brody, Compact manifolds and hyperbolicity, Trans. Amer. Math. Soc. 235 1978, 213-219.

[4] H. Cartan, Sur les systèmes de fonctions holomorphes à variètès lacunaires et leurs aapplications , Ann. Sci. Ecole Norm. Sup. 45 1928, p. 255-346.

[5] J. Duval, Around Brody lemma, arXiv preprint arXiv:1703.01850, 2017.

[6] B. Davis, Picard's theorem and Brownian motion, Trans. Amer. Math. Soc. 213 1975, 353-362.

[7] J. Duval, Un théorème de Green presque complexe, Annales de linstitut Fourier 54 no 7 2004, 2357-2367

[8] M. GREEN, Some Picard theorems for holomorphic maps to algebraic varieties, Amer. J. Math. 971975 , pp. 43-75.

[9] M. GREEN, Holomorphic maps into complex projective space omitting hyperplanes, Trans. Amer. Math. Soc., 169 1972, pp. 89-103.

[10] S. Lang, Introduction to Complex Hyperbolic Spaces, New York, Inc. in 1987, pp. 196

[11] M. Zaidenberg, Hyperbolic surfaces in $\mathbf{P}^{3}$ : examples, arXiv preprint math/0311394 2003.

${ }^{1}$ UNIVERSITY OF MONASTIR,

INSTITUT PRÉPARATOIRE AUX ETUdes D'INGÉNIEUR DE MONASTIR, MONASTIR, TUNISIA

Email address: fathi.haggui@gmail.com

2 UNIVERSITY OF MONASTIR,

FACULTY OF SCIENCES OF MONASTIR,

MONASTIR, TUNISIA

Email address: jalled.abdessami 90 @gmail.com

Received: 10.2.2021

Revised: 7.5.2021

Accepted: 29.5.2021 